\title{
Surgical margins in head and neck cancer: A contemporary review
}

\author{
Michael L. Hinni, MD, ${ }^{1}$ Alfio Ferlito, MD, DL0, DPath, FRCSEd ad hominem, FRCS (Eng,Glasg, Ir) ad eundem, FDSRCS ad hominem, FHKCORL, FRCPath, \\ FASCP, IFCAP, ${ }^{2}$ Margaret S. Brandwein-Gensler, MD, ${ }^{3}$ Robert P. Takes, MD, PhD, ${ }^{4}$ Carl E. Silver, MD, ${ }^{5}$ William H. Westra, MD, ${ }^{6}$ Raja R. Seethala, MD, ${ }^{7}$ \\ Juan P. Rodrigo, MD, PhD, ${ }^{8,9}$ June Corry, MD, FRACP, FRANZCR, ${ }^{10}$ Carol R. Bradford, MD, ${ }^{11}$ Jennifer L. Hunt, MD, ${ }^{12}$ Primož Strojan, MD, PhD, ${ }^{13}$ \\ Kenneth 0. Devaney, MD, JD, FCAP, ${ }^{14}$ Douglas R. Gnepp, MD, ${ }^{15}$ Dana M. Hartl, MD, PhD, ${ }^{16,17}$ Luiz P. Kowalski, MD, PhD, ${ }^{18}$ \\ Alessandra Rinaldo, MD, FRCSEd ad hominem, FRCS (Eng, Ir) ad eundem, FRCSGlasg, ${ }^{2}$ Leon Barnes, MD, FASCP, FCAP'
}

${ }^{1}$ Department of Otolaryngology-Head and Neck Surgery, Mayo Clinic, Phoenix, AZ, ${ }^{2}$ ENT Clinic, University of Udine, Udine, Italy, ${ }^{3}$ Surgical Pathology Section, Division Anatomic Pathology, University of Alabama at Birmingham, Birmingham, AL, ${ }^{4}$ Department of Otolaryngology-Head and Neck Surgery, Radboud University Nijmegen Medical Center, Nijmegen, The Netherlands, ${ }^{5}$ Departments of Surgery and Otolaryngology-Head and Neck Surgery, Albert Einstein College of Medicine, Montefiore Medical Center, Bronx, NY, ${ }^{6}$ Departments of Pathology and Otolaryngology-Head and Neck Surgery, The Johns Hopkins Medical Institutions, Baltimore, MD, ${ }^{7}$ Department of Pathology and Laboratory Medicine, University of Pittsburgh, Pittsburgh, PA, ${ }^{8}$ Department of Otolaryngology, Hospital Universitario Central de Asturias, Oviedo, Spain, ${ }^{9}$ Instituto Universitario de Oncología del Principado de Asturias, Oviedo, Spain, ${ }^{10}$ Division of Radiation Oncology, Peter MacCallum Cancer Centre, Melbourne, Victoria, Australia, ${ }^{11}$ Department of OtolaryngologyHead and Neck Surgery, University of Michigan, Ann Arbor, MI, ${ }^{12}$ Department of Pathology, University of Arkansas for Medical Sciences, Little Rock, AR, ${ }^{13}$ Department of Radiation Oncology, Institute of Oncology, Ljubliana, Slovenia, ${ }^{14}$ Department of Pathology, Allegiance Health, Jackson, Ml, ${ }^{15}$ Department of Pathology, Brown University School of Medicine, Rhode Island Hospital, Providence, RI, ${ }^{16}$ Department of Otolaryngology-Head and Neck Surgery, Institut Gustave Roussy, Villejuif, France, ${ }^{17}$ Laboratoire de Phonétique et de Phonologie, Sorbonne Nouvelle, Paris, France, ${ }^{18}$ Department Otorhinolaryngology-Head and Neck Surgery, Centro de Tratamento e Pesquisa Hospital do Cancer A.C. Camargo, São Paulo, Brazil.

Accepted 28 May 2012

Published online 3 September 2012 in Wiley Online Library (wileyonlinelibrary.com). DOI 10.1002/hed.23110

\begin{abstract}
Adequate resection margins are critical to the treatment decisions and prognosis of patients with head and neck squamous cell carcinoma (HNSCC). However, there are numerous controversies regarding reporting and interpretation of the status of resection margins. Fundamental issues relating to the basic definition of margin adequacy, uniform reporting standards for margins, optimal method of specimen dissection, and the role of intraoperative frozen section evaluation, all require further clarification and standardization. Future horizons for margin surveillance offer the possible use of novel methods such as
\end{abstract}

"molecular margins" and contact microscopic endoscopy, However, the limitations of these approaches need to be understood. The goal of this review was to evaluate these issues to define a more rational, standardized approach for achieving resection margin adequacy for patients with HNSCC undergoing curative resection. (c) 2012 Wiley Periodicals, Inc. Head Neck 35: 1362-1370, 2013

KEY WORDS: head neck cancer, pattern of invasion, surgical margins, frozen section, molecular factors

\section{INTRODUCTION}

Head and neck squamous cell carcinoma (HNSCC) may be treated by surgery, radiation therapy, systemic therapy, or combinations of these modalities. In general, local tumor control is best achieved by complete surgical excision with "adequate" resection margins. Although the concept of margin adequacy may seem straightforward, considerable confusion surrounds the definition of adequate margins. There are also sizeable differences regarding the extent of surgery performed, and the method of determining margin status. Thus achieving resection margin adequacy is highly "operator dependent" with respect to surgeons and pathologists. The practice of quantifying resection margin distance on the pathology report has not been universally adapted. Unfortunately,

*Corresponding author: A. Ferlito, ENT Clinic, University of Udine, Piazzale S. Maria della Misericordia, I-33100 Udine, Italy. E-mail: a.ferlito@uniud.it.

This article was written by members and invitees of the International Head and Neck Scientific Group. nonquantifiable, ambiguous terms such as "close margins" are still being used. Worse yet, margins may be deemed "negative" or "free" when, in fact, they are inadequate. This lack of clarity is unfortunate because adjuvant treatment decisions and patient outcomes are profoundly influenced by the completeness of tumor removal. The surgical management of HNSCC would greatly benefit from a more accurate and standardized approach to resection margins.

A refined, standardized approach to margin surveillance should ideally account for various anatomic and tumor factors such as the 3-dimensional aspects of tumor extension and pathologic factors such as the pattern of tumor invasion. Changing surgical practices impact the optimal method of margin surveillance. Open or transoral en-bloc resections are amenable to comprehensive "specimendriven" tumor mapping and margin surveillance, as will be discussed. However, the same approach may not be feasible for endoscopic or piecemeal laser microscopic resections, where multiple tumor fragments can add complexity to the surgeon/pathologist team.

More sophisticated imaging and optical techniques are now being developed that may allow surgeons, before 
and during surgery, to pinpoint tumor boundaries of primary carcinomas more precisely. This also has the potential to impact margin surveillance. These optical techniques, however, are largely limited to the surface mucosa and cannot assess deep resection margins. The ability to detect genetically damaged, albeit phenotypically normal mucosal cells has been proposed as the basis for novel molecular assays as a form of margin assessment, which has been termed "molecular margins." Although this represents, theoretically, a promising avenue for upper aerodigestive tract tumor mapping, there is little clinical experience with this method, and, once again, this strategy cannot address deep resection margins.

The goal of this review was to summarize the debates regarding the above issues, review the literature, provide evidence or a rationale for consensus and standardization, and finally, where possible, suggest a practice standard for margin surveillance.

\section{Definition and prognostic impact of positive margins}

Despite an overwhelming consensus that failure to eradicate tumor at the primary site is the single largest cause of death for patients with HNSCC, ${ }^{1}$ there is no universal definition of an inadequate resection margin. In a survey of the American Head and Neck Society, ${ }^{2}$ members were asked how they evaluate and define tumor margins. The most common response for distance of a clear pathologic margin was $>5 \mathrm{~mm}$ on microscopic evaluation. Margins containing carcinoma "in-situ," but not "dysplasia," were considered positive by most respondents. When the results of initial frozen section margins are positive and further supplemental resection margin results are negative, then $90 \%$ of respondents considered the final resection margin status as negative. This survey underscored the fact that uniform criteria defining adequate surgical margins are lacking among practicing head and neck surgeons. ${ }^{2}$

Most will agree that resection margins should be assessed as "perpendicular margins" (Figure 1). Histologic sections should be taken from the tumor invasive front to the nearest surgical resection edge and measured in millimeters. This is in distinction to parallel, en-face margins, which assess greater surface area, but do not allow for the measurement of margin distance. Most studies classify margins as either (1) positive, that is, tumor "cutthrough"; (2) close; or (3) negative margin, with varying definitions of close and negative. The crux of the controversy revolves around the necessary distance between the carcinoma and "the surgeon's knife," be it stainless steel, electrocautery, or laser. Most studies that specifically define margin distance use a somewhat arbitrary definition of $\geq 5 \mathrm{~mm}$ to define margin adequacy. ${ }^{3-9}$ Many studies demonstrate improved outcomes with adequate (negative) margins. For instance, Chen et $\mathrm{al}^{4}$ reported on 270 consecutive patients with carcinoma of the oral cavity, oropharynx, hypopharynx, and larynx, using a defined 5$\mathrm{mm}$ margin standard. Locoregional recurrence and 5-year disease-free survival rates were $55 \%$ and $7 \%$, versus $17 \%$ and $39 \%$, for patients with inadequate versus adequate margins, respectively. Similarly, Loree and Strong 5 reported the outcome for 398 consecutive patients with oral cancer, using a defined 5-mm standard for margins.

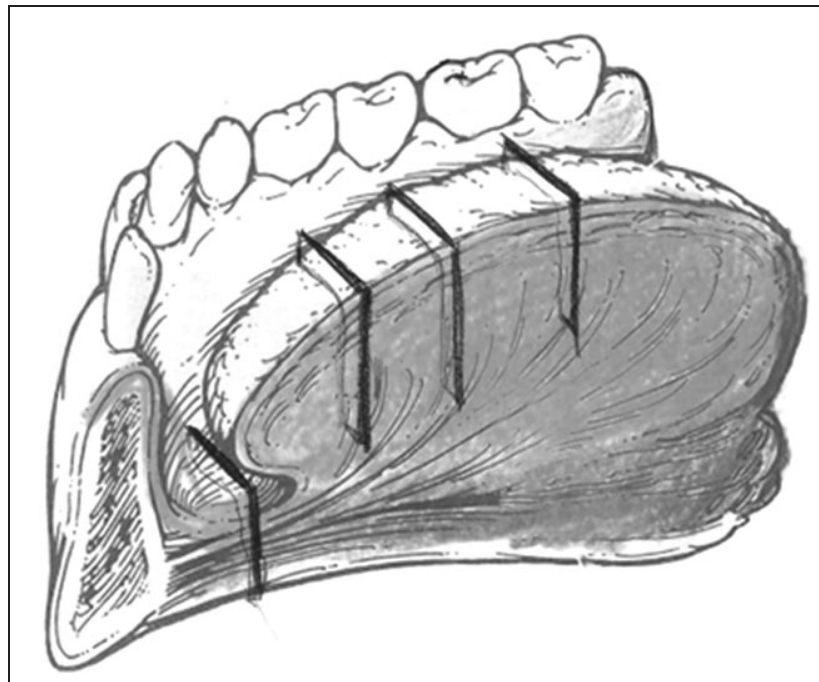

FIGURE 1. Combined mandibular and lateral tongue resection demonstrating mapping of different margin planes (eg, anterior floor of mouth, tongue root, midline deep tongue). The perpendicular planes represent the direction of cuts the pathologist makes into the specimen at the time of specimen "hand-off." Modified from Surgical Pathology Dissection-An Illustrated Guide, 2nd edition, W Westra, R Hruban, T Phelps, C Isacson, Springer, New York 2003, with permission.

Locoregional recurrence and 5-year overall survival rates were $30 \%$ and $52 \%$ versus $18 \%$ and $60 \%$ for patients with inadequate versus adequate margins, respectively. Liao et $\mathrm{al}^{10}$ reviewed 827 consecutive patients with oral cancer and examined the impact of different cut-off values for resection margins $(\leq 3 \mathrm{~mm}$ to $\leq 11 \mathrm{~mm}$, in $1-\mathrm{mm}$ intervals) by hazard ratio and $95 \%$ confidence intervals. This large-scale study is the only one to address the issue of "optimum" margin distance. On multivariate analysis, they demonstrated that resection margins of $\leq 7 \mathrm{~mm}$ were significantly associated with decreased local disease control. $^{10}$

Table 1 summarizes publications that define resection margin standards, and their associated outcome. ${ }^{4-15}$ Interestingly, not all groups demonstrate that adequate resection margins are associated with better outcome as compared with patients with inadequate margins. $8,9,11,12$ Why would some "inadequate" resections be associated with better outcomes than other "inadequate" resections? We hypothesize that the more optimal the surgical resection and the more optimal the intraoperative margin assessment, the better the outcome for patients with "inadequate" margins. Narrowing the outcome gap between patients with adequate versus inadequate margins would be seen as a lack of significant difference in outcome between these 2 groups. We speculate that the degree of "inadequate" margins reported in the studies by Chen et al, ${ }^{4}$ Loree and Strong, ${ }^{5}$ and Garzino-Demo et $\mathrm{al}^{6}$ may have been underestimated because of the significantly worse outcomes for these patients. Without question there are significant institutional and practice differences between institutions, making scientific comparisons difficult regarding margin assessment.

\section{Laryngeal resection margins: A unique situation}

The treatment of laryngeal cancer includes the additional consideration of balancing voice preservation with 
TABLE 1. Summary of publications that define resection margin standards and their associated outcome.

\begin{tabular}{|c|c|c|c|c|c|c|}
\hline Author & Year & $\begin{array}{l}\text { Sites } \\
\text { (all stages, } \\
\text { unless otherwise } \\
\text { indicated) }\end{array}$ & Patients & $\begin{array}{l}\text { Margin } \\
\text { definition }\end{array}$ & $\begin{array}{l}\text { Locoregional } \\
\text { recurrence } \\
\text { (inadequate versus } \\
\text { adequate margins) }\end{array}$ & $\begin{array}{l}\text { Survival (inadequate } \\
\text { versus adequate } \\
\text { margins) }\end{array}$ \\
\hline Chen et al ${ }^{4}$ & 1987 & $\begin{array}{l}\text { Oral, oropharyngeal, } \\
\text { laryngeal, } \\
\text { hypopharyngeal }\end{array}$ & 270 & $5 \mathrm{~mm}$ & $55 \%$ vs $17 \%$ & $\begin{array}{l}7 \% \text { vs } 39 \% \text { 5-year } \\
\text { disease-free survival }\end{array}$ \\
\hline Loree and Strong $^{5}$ & 1990 & Oral & 303 & $5 \mathrm{~mm}$ & $30 \%$ vs $18 \%$ & $52 \%$ vs $60 \% 5$ year OS \\
\hline El-Husseiny et $\mathrm{al}^{7}$ & 2000 & Tongue & 66 & $5 \mathrm{~mm}$ & $0 \%$ vs $63 \%$ DFS & $21 \%$ vs $72 \%$ \\
\hline de Visscher et al ${ }^{14}$ & 2002 & Lip & 72 & $3 \mathrm{~mm}$ & $\begin{array}{l}8 \text { patients had } \\
\text { inadequate margins. } \\
\text { LR developed in } 1 \text { of these } \\
8 \text { patients, plus } 1 \text { patient } \\
\text { with adequate margins. }\end{array}$ & \\
\hline Sutton et al ${ }^{15}$ & 2003 & Oral & 200 & $5 \mathrm{~mm}$ & $\begin{array}{l}55 \% \text { positive vs } 33 \% \\
\text { close vs } 12 \% \text { negative }\end{array}$ & $\begin{array}{l}0 \% \text { positive vs } 26 \% \\
\text { close vs } 54 \% \text { negative } \\
\text { alive and disease-free }\end{array}$ \\
\hline McMahon et al ${ }^{13}$ & 2003 & $\begin{array}{l}\text { Oral and } \\
\quad \text { oropharyngeal }\end{array}$ & 332 & $10 \mathrm{~mm}$ & $\begin{array}{l}\text { Margin status associated } \\
\text { with LR and DSS on } \\
\text { univariate analysis but } \\
\text { not multivariate analysis }\end{array}$ & \\
\hline Amaral et al ${ }^{8}$ & 2004 & $\begin{array}{l}\text { Oral, } \\
\quad \text { Stage } 1 / \| l\end{array}$ & 188 & $5 \mathrm{~mm}$ & & $66 \%$ vs $73 \%$ DFS \\
\hline Weijers et al ${ }^{9}$ & 2004 & Oral & 68 & $5 \mathrm{~mm}$ & $\begin{array}{l}6.6 \% \text { vs } 7.9 \% \text { (adequate versus } \\
\text { close, positive margins excluded) }\end{array}$ & \\
\hline Kademani et al ${ }^{11}$ & 2005 & Oral & 233 & $2 \mathrm{~mm}$ & & $\begin{array}{l}\text { "Positive margins did } \\
\text { not influence survival" }\end{array}$ \\
\hline Brandwein-Gensler et al ${ }^{12}$ & 2005 & $\begin{array}{l}\text { Oral and } \\
\quad \text { oropharyngeal }\end{array}$ & 168 & $5 \mathrm{~mm}$ & $\begin{array}{l}23 \% \text { vs } 13 \% \text { (adequate versus } \\
\text { close margins, positive } \\
\text { margins excluded) }\end{array}$ & \\
\hline Garzino-Demo et $\mathrm{al}^{6}$ & 2006 & Oral & 245 & $5 \mathrm{~mm}$ & & $48 \%$ vs $65 \% 5$ year OS \\
\hline Liao et $\mathrm{al}^{10}$ & 2008 & Oral & 827 & \multicolumn{2}{|c|}{$\begin{array}{l}\text { This group examined the impact of } \\
\text { different resection margin cut-off } \\
\text { values for resection margins ( } \leq 3 \mathrm{~mm} \\
\text { to } \leq 11 \mathrm{~mm} \text {, in } 1 \mathrm{~mm} \text { intervals) by } \\
\text { hazard ratio and } 95 \% \text { confidence intervals. } \\
\text { On multivariate analysis, resection margins } \\
\text { of } \leq 7 \mathrm{~mm} \text { were significantly associated with } \\
\text { decreased local disease control. }\end{array}$} & \\
\hline
\end{tabular}

Abbreviations: OS, overall survival; DFS, disease free survival; LR, local recurrence; DSS, disease specific survival.

local disease control. There is a long-standing consensus that glottic resection margins may be as limited as 1 to 2 $\mathrm{mm}$ and still be considered adequate ${ }^{16,17} \mathrm{~T} 1$ glottic carcinomas may be treated by transoral laser surgery; several retrospective studies have demonstrated that close or positive margins had no impact on local recurrence. ${ }^{8-22}$ This finding may be due to the thermal laser effects at the resection margins, the inherent excellent outcome of T1 glottic carcinomas, or possibly the small size of the specimens and the shrinkage during the technical workup for pathologic analysis.

Because of the anatomic constraints of the larynx, many laryngeal studies use a defect-driven approach to margin surveillance, in which the margin biopsy specimen is taken from the cut border of tissue remaining in the patient. The surgeon selects the tissues to be evaluated for margin status, and margins are evaluated as either "positive" or "negative." In this context, retrospective studies including higher stage glottic cancers have demonstrated that positive margins are significantly associated with decreased local control. Peretti et $\mathrm{al}^{23}$ reported on 595 patients with Tis-T3 glottic carcinomas and demon- strated a modest, but significant, association with positive, "cut-through" deep margins and decreased local disease control. Crespo et $\mathrm{al}^{24}$ reported on 40 patients with $\mathrm{T} 1$ and T2 glottic carcinomas and also demonstrated that positive "cut-through" margins were significantly related to local recurrence. Blanch et $\mathrm{al}^{25}$ reported on 107 patients with T2/T3 anterior commissure glottic carcinomas and demonstrated that positive "cut-through" margins were significantly associated with increased local failure. Spector et $\mathrm{al}^{26}$ reported on 42 patients with $\mathrm{T} 3 \mathrm{~N} 1$ glottic cancers treated by total laryngectomy and reported that positive "cut-through" margins were significantly associated with decreased disease-free survival, regardless of whether adjuvant radiation therapy had been administered.

Gallo et $\mathrm{al}^{27}$ reported on 253 patients treated by supracricoid partial laryngectomy, which included 42 patients with supraglottic cancers. Here, they defined "positive margins" as cancer or dysplasia $<5 \mathrm{~mm}$, although they did not further clarify the degree of dysplasia. Multivariate analysis demonstrated that positive margins remained significantly predictive of local disease control. Bradford et $\mathrm{al}^{28}$ reported on 159 surgically treated patients with 
stage III/IV laryngeal cancer, including 97 patients with supraglottic cancers, as part of the Department of Veterans' Affairs Laryngeal Cancer Study. Patients with close $(<5 \mathrm{~mm})$ or positive "cut-through" margins received significantly higher doses of postoperative radiation. Patient survival percentages were $50 \%$ for clear margins, $57 \%$ for close margins, and $27 \%$ for involved margins $(p=$ .286). Although the higher radiation doses may have minimized the survival differences between patients with close and negative margins, the data suggest that highdose postoperative radiation does not eliminate the benefit of obtaining widely clear surgical margins. A retrospective study of 653 patients limited to supraglottic cancers, all stages, treated by either open supraglottic laryngectomy or total laryngectomy, demonstrated that positive margins were significantly associated with decreased disease-free survival. ${ }^{29}$ On balance it seems clear that very narrow margins are adequate for the glottic larynx, but wider margins are necessary for carcinomas of transglottic or supraglottic locations.

\section{Margin inadequacy: Deep tissues versus mucosa}

Surgeons are more likely to encounter difficulties in achieving adequate margins for deep connective tissue planes as compared with mucosal margins. In a review of 301 patients with oral and oropharyngeal cancers resected with curative intent, deep soft tissue margins were involved in $87 \%(61 / 70)$ of resections with inadequate margins. In contrast, resections with inadequate mucosal margins alone were rare, seen in $16 \%$ (11/70) of cases. Hence, less than $2 \%$ of 301 resections had inadequate margins solely on the basis of mucosal margins. ${ }^{30}$ This is especially relevant in the context of newer approaches such as "molecular margins" or confocal microscopic endoscopy, which assess only mucosal surfaces. Anatomic constraints may also limit the surgeon's ability to achieve adequate deep resection margins. Another factor explaining the higher incidence of inadequate deep soft tissue margins may be that on the mucosal surface the tumor is visible whereas during excision of the tumor in the soft tissues the tumor is not visible but only palpable when one attempts an "en bloc" resection (piecemeal resections may mitigate this risk). Moreover, factors such as perineural and lymphovascular invasion and infiltrative growth patterns are more likely to be relevant in the cause of inadequate deep soft tissue margins. These factors will be discussed later in this review.

\section{Intraoperative margin surveillance: The en-bloc approach}

An interesting survey of 200 pathologists in 2006 reported that most surgeons send small tissue fragments to pathologists as a means of HNSCC margin assessment. ${ }^{31}$ Although this does have speed advantages and more control in terms of "true margin" accuracy in situ, particularly with respect to more spatially complex specimens, this approach can certainly underestimate the real status of resection margins. We advocate that intraoperative resection margin surveillance for en-bloc resections should be "specimen driven," rather than "defect driven," as defined above. Because many of the older publications regarding margins never specifically discussed the manner of margin assessment, it is likely that the reported literature is based on a mixture of both "specimen-driven" and "defect-driven" approaches.

Small resections, such as wedge resections from the tongue, are easily oriented by sutures. Large or complicated resections may require that the surgeon personally hand off the specimen to the pathologist and participate in specimen mapping. The first 10 minutes of pathologist/ surgeon interaction after specimen hand-off yields the most valuable information. Whenever possible, face-toface interaction is recommended. The approach is identical regardless of whether the en bloc specimen was harvested via open surgery or transoral robotic surgery. Translating a complex 3-dimensional resection specimen into a comprehensive 2-dimensional map can be challenging. It is recommended to document the process, either with a diagram or a digital image that includes labels of the anatomic sites. Some surgeons ink the different resection margin planes with different colors and keep careful records that are an additional component of mapping (eg, red $=$ superior). Different inking is particularly important when a section will include more than 1 margin (ie, deep and ventral) on the slide. Resection margins are defined as any tissue plane where the surgeon's knife meets the patient. The surgeon identifies intraoperative nonmargin tissue tears or cuts. Rather than avoiding inking these surfaces, we recommend inking these tears or cuts in a new specified color (eg, yellow = tissue tear).

After orienting, mapping, and inking the resection specimen, the pathologist surveys the resection planes by cutting into the specimen at 2- to 4-mm intervals perpendicular to the resection margin plane (Figure 1). This gross assessment yields important preliminary information. The surgeon may elect to return to the defect at this time to harvest more tissue on the basis of the gross examination result, as slides are being processed. This is followed by microscopic examination, which further refines the information. An easy method for pathologists to histologically measure distance accurately in millimeters is to print out a metric ruler on acetate paper (see http://www.vendian.org/mncharity/dir3/paper_rulers/) and overlay the clear ruler on the slide.

A recent review of intraoperative margin surveillance for 61 patients undergoing supracricoid laryngectomy demonstrated positive margins in $11 \%$ of resections. ${ }^{32}$ Harvesting supplemental resection margins successfully addressed initial positive margins in all but 1 patient, who required total laryngectomy. All of the positive margins were found at the ipsilateral posterior and inferior margin planes; the authors recommend particular sampling of these margins. Of note, the thermal effects of both laser and electrocautery on tissues have reportedly introduced problems in interpreting the histologic study results. However, permanent histologic study confirmed the frozen section results in $94.8 \%$ of the interventions in 1 study. The authors concluded that intraoperative margin surveillance is reliable for laser-assisted procedures, with a high negative predictive value when performed by an experienced team. ${ }^{32}$

It is common practice for surgeons to address close margins at the time of frozen section by harvesting wide 
swatches of "supplemental margins" from the corresponding regions of the defect. A combined approach of "specimen-driven" and "defect-driven" margin surveillance may be necessary for some spatially complex specimens, because many landmarks retract or are lost on a main specimen. "Defect disorientation" can limit the surgeon's ability to accurately relocate the site of inadequate margin planes. This disorientation can be anticipated and solved by marking the various margin points within the surgical defect by use of clips or sutures, before bringing the resection specimen to the frozen section suite. ${ }^{33}$

A false-negative frozen section report is usually the result of sampling error; carcinoma may be absent in the actual frozen section slide but appears in the permanent sections after deeper cutting into the formalin-fixed paraffin-embedded block. Two groups have demonstrated that extensive histologic evaluation with multiple levels at the time of frozen sections decreases the false-negative rate for intraoperative margin assessment. ${ }^{34,35}$

\section{Intraoperative margin surveillance: The multiblock approach}

Intraoperative margin assessment is especially important in conservation laryngeal surgery where, because of the small size and high complexity of the larynx, close surgical margins are often inevitable. Endoscopic laryngeal resection with $\mathrm{CO}_{2}$ laser is replacing the external laryngeal approach. This technique changes the paradigm of margin surveillance, because en-bloc resection may be replaced by multiblock resection. With "transoral laser microsurgery," the surgeon will deliberately laser through tumor to remove manageable sections of tumor through the necessary narrow confines of an exposing laryngoscope. Transoral laser microsurgery is applicable to head and neck cancers at all head and neck locations within the upper aerodigestive tract. This technique has the advantage of allowing the surgeon to map the 3-dimensional depths of the tumor under high powered operative microscopy visualization. Many laser surgeons will ink the edge of each tumor section on the edge to prevent fragment disorientation during evaluation by frozen section. Particularly where the glottis is concerned, "defectdriven" margin assessment should be avoided because the extra tissue taken can unnecessarily harm subsequent vocal function. There may be situations, however, where defect-driven margin sampling is unavoidable. In this setting, margins must be reported as either positive or negative.

Patients with laryngeal cancer with positive margins on permanent pathology often undergo second operations to address these margins; residual cancer may be identified in up to $31 \%$ of second procedures. ${ }^{18}$ Perhaps the largest experience with second look surgeries, for laryngeal, as well as oral resections, is reported by Jäckel et al. ${ }^{36}$ and Steiner et al. ${ }^{37,38}$ As a result, in part, of the physical separation of the pathology laboratory and the surgical suite, second looks were initially required in more than a third of the patients. In spite of this, when positive margins were identified on permanent pathology, clear margins were ultimately obtained during a "second-look" proce- dure in the vast majority of patients. This resulted in no measurable difference in local control or overall survival.

In conclusion, achieving adequate resection margins at initial surgical treatment is good practice. This has the potential to eliminate the need for additional surgery or adjuvant therapy. Most authors agree that intraoperative resection margin surveillance is valuable; it requires close cooperation and good communication between surgeon and pathologist. Achieving adequate resection margins can be expected to impact local control and may affect overall survival.

\section{Tissue shrinkage}

No discussion regarding resection margins is complete without raising the issue of tissue shrinkage. Tissue under tension will contract after resection; thus surgeons lament that the margins shrink and the surgical defect expands. Canine studies have demonstrated that tongue mucosa contracts more than deep tongue muscle after resection $(24.8 \%$ vs $20.9 \%))^{39}$ The only study to address margin shrinkage in patients with head and neck cancer appears to have measured only mucosal contraction, which was on the order of $20 \%$ to $25 \% .^{40}$ These figures provide some rough "rule of thumb" for surgeons. Surgeons need to place $20 \%$ to $25 \%$ more tissue between the tumor and "the blade" to achieve a particular margin distance.

Tissue formalin fixation and paraffin embedding will also add to tissue shrinkage, but to a lesser degree, on the order of $10 \%$. In the experience of one of the authors (M.B.G.), who compared margin distances at the time of gross examination in the patient, in the frozen section slide and in the corresponding permanent section slide, tissue shrinkage has minimal overall effect on the final margin status. Margin status discrepancies between frozen and permanent sections are more likely due to increased sampling rather than tissue shrinkage.

\section{Bone margins}

Because of its high mineral content and need for decalcification, bone is generally not amenable to rapid tissue analysis. Osseous margins have therefore been traditionally determined before surgery by viewing imaging studies. The need for a more definitive and immediate histologic intraoperative evaluation of bone margins for residual tumor is obvious and has been the subject of several studies.

Forrest et $\mathrm{al}^{41}$ evaluated the bone margins during surgery of 29 adult patients with biopsy-proven carcinoma who underwent full-thickness mandibular resection. Using a curette, the proximal $1 \mathrm{~cm}$ of cancellous (not cortical) bone was sampled from the mandibular stump (not from the resected specimen). With standard frozen section technique (cryostat and $4-\mu \mathrm{m}$-thick sections), this method was able to predict the final pathologic surgical margin in $97 \%$ of cases. Mahmood et $\mathrm{al}^{42}$ examined the bony resection margins of 7 patients undergoing segmental resection of the mandible for squamous cell carcinoma. Instead of frozen section, they used intraoperative cytologic assessment of bone marrow scrapings to predict margin status. The procedure proved to be easy, cost-effective, and 
reliable. All specimens examined correlated with the final results obtained after conventional bone decalcification.

Because of its hardness, cortical bone cannot be sectioned without decalcification before histologic evaluation. Some recent procedures have been developed that circumvent this issue and allow cortical bone to be examined histologically at the time of surgery. Oxford and Ducic $^{43}$ describe their technique in which a 4- to 5-mm sharpened, curved osteotome is used to obtain thin, translucent sections of cortical bone margins derived from the resected specimen. Because the sections are so thin, they require no decalcification and can therefore be examined by routine frozen section. The procedure, according to the authors, is highly sensitive $(89 \%)$ and specific $(100 \%)$ with respect to findings on permanent decalcified sections. Wysluch et $\mathrm{al}^{44}$ describe another procedure for examining cortical bone during surgery. They use a trephine drill to obtain 1 or more $5-\mathrm{mm}$ length cores of bone from the resected margins. A contact gel is applied to the cores, which are then cooled on a carrier plate. Cryostat sections can then be obtained, and the slides, after removal of the gel, are ready for staining and evaluation. According to the authors, the entire processing time including diagnostic examination averages 30 minutes. In this study the intraoperative frozen section evaluation and the final pathologic diagnosis were in agreement in $94 \%$ of cases.

Other, more sophisticated means of evaluating bone margins at the time of surgery have been proposed, such as intraoperative microwave processing and elastic scattering spectroscopy. ${ }^{45,46}$ These procedures, however, are often labor-intensive and require expensive instruments or specialty-trained personnel.

Regardless of which procedure is used to obtain the specimen, intraoperative interpretation of bone margins can be challenging. There is often considerable artifactual distortion of the specimen, the background is usually hemorrhagic and contaminated with bone dust from the drill or saw, and normal immature hematopoietic precursors may at times cause concern or even be mistaken for tumor cells. However, despite these challenges, we believe that bony margins can and should be routinely assessed during head and neck cancer operations where bone is involved by tumor.

In contrast to bone, cartilage, unless heavily calcified or ossified, can usually be examined by standard frozen section without prior preparation. A common technical problem, however, is the tendency of the cartilage to "float" off the slide during staining. This can be diminished by precoating the slide with albumen or gelatin, which serves as an adhesive. Irrespective of these modest challenges, we believe that bony and cartilaginous margins can and should be routinely assessed during head and neck cancer operations.

\section{Use of imaging and optical techniques}

Contact endoscopy ("in vivo" microscopic examination of laryngeal mucosa with a rigid endoscope) has been evaluated as an auxiliary method to accurately distinguish between healthy mucosa, dysplasia, and invasive carcinoma during surgery. Contact endoscopy has the advantage over frozen section histopathologic study in that it is noninvasive and provides information on microscopic diagnosis and laryngeal lesion margins. One study reported a sensitivity of $80 \%$, a specificity of $100 \%$, and an accuracy of $93 \%$ for contact endoscopy in the diagnosis of malignancy. ${ }^{47}$ Another study examined contact endoscopy for frontolateral approach of glottic cancers; the lesion and its limits were carefully defined, the surgical margins were established under the rigid telescope, and the tumor margins were studied under contact endoscopy after methylene blue staining. Frozen section examination of the margins was performed, and the histopathologic analysis was compared with the surgical and endoscopic findings. The surgical margins were free of disease in all cases, and there was a $100 \%$ correlation with the histopathologic examination, supporting the utility of contact endoscopy. ${ }^{48}$ Another recent development that may allow for more accurate surgery is the visualization of tumor cells by fluorescent labeling. ${ }^{49}$ With these techniques of image-guided surgery, tumor extension and deep, as well as mucosal, resection margins may be assessed more accurately during surgery. On the other hand, simply adopting an anatomic approach to cancer surgery by studying preoperative CT or MRI scans may also reduce the incidence of positive margins. In 1 report, experienced surgeons identified positive margins $28 \%$ of the time. This number dropped to $5 \%$ after the advent of routine preoperative CT study. ${ }^{50}$ In another recent study of carcinoma of the maxilla, more than one third of 69 patients were found to have positive margins. When compared by careful imaging, both dorsal and cranial tumor extension was significantly associated with positive margins. The margin status when positive was linked to a doubling in the risk of death. ${ }^{51}$

\section{Histopathologic and molecular factors}

The biologic behavior or aggressiveness of individual cancers clearly impacts the ability to achieve local control. Broder's "grading system," on the basis of the degree of tumor keratinization, was perhaps the first attempt at microscopically predicting tumor behavior. ${ }^{52}$ Tumor lymphovascular emboli and perineural invasion are also prognostically significant. ${ }^{53}$ However, multifactorial grading algorithms were found to be more predictive of outcome than 1 single parameter alone. ${ }^{54,55}$ This served as the forerunner for a more complex risk model proposed by Brandwein-Gensler et al. ${ }^{12,56}$ The inability to obtain adequate resection margins is also an indicator of inherent tumor aggressiveness. The pattern of tumor invasion impacts the ability to clear deep resection margins. Tumor worst patterns of invasion (WPOI) can be classified as aggressive if carcinomas contain either small tumor islands ( $\leq 15$ cells) that are separate from but close to the main tumor mass (WPOI type 4 ), or worse yet, if the tumor satellites are dispersed (WPOI type 5). ${ }^{12,56}$ These WPOI have been validated as significant prognosticators of outcome. ${ }^{56}$ A number of histologic phenotypes can be seen with WPOI type 5, including dispersed growth via lymphovascular tumor emboli, and dispersed growth via perineural invasion. Both aggressive WPOI are significantly associated with inadequate margins at deep tissue planes. ${ }^{12}$ However, there is no literature at this time to 
support the relevance of these findings with respect to management of margins, specifically whether high-risk tumors require greater margin distances than low-risk under these scoring schemes.

The concept of field cancerization is important in any discussion of surgical margins and mucosal margins in particular. First described in 1953, this somewhat controversial idea is that HNSCCs arise from a "field" of genetically damaged or "condemned" mucosa. ${ }^{57}$ Recent observations have supported the view that the epithelium of the upper respiratory tract may become populated by tracts of genetically damaged cells, which lack histopathologic evidence of dysplasia. ${ }^{58}$ The presence of morphologically intact but genetically damaged cells would seem to explain certain distressing patterns of HNSCC behavior such as local tumor recurrence after seemingly "complete" surgical resection with "negative" margins. A growing lack of confidence in the pathologist's ability to recognize the presence and extent of the neoplastic process in patients at risk for HNSCC has accelerated a search for novel biomarkers in the recognition and treatment of HNSCC, particularly in the evaluation of surgical margins. Indeed, the presence of genetically altered cells can be detected in histologically normal mucosal margins with a variety of strategies for detecting genetic alterations including TP53 mutations, ${ }^{59}$ loss of heterozygosity, ${ }^{60}$ promoter hypermethylation, ${ }^{61,62}$ eIF4E proto-oncogene overexpression, ${ }^{63}$ and mitochondrial DNA mutations. ${ }^{64}$ Importantly, the presence of these genetically damaged cells has been shown to predict local tumor recurrence in patients who have undergone tumor resections with histologically clear margins. Although molecular margin assessment has been studied in the literature with some promising early evidence, this type of analysis is currently investigational and not used widely in clinical practice.

Although a rising percentage of HNSCCs, particularly those arising from the oropharynx, are associated with high-risk human papillomavirus (HPV), an HPV detection strategy has not yet been implemented as a practical tool for assessing margin status for the resection of HPV-positive tumors. First, these HPV-associated squamous cell carcinomas do not appear to arise in a widespread field of HPV infected mucosa. ${ }^{65}$ In effect, HPV detection would not be very useful in determining the extent of disease in histologically normal mucosa. Second, most oropharyngeal HPV-associated squamous cell carcinomas are not treated surgically.

There are practical, financial, and even ethical conflicts that emerge from discussions regarding the utility of molecular margins that currently add time and expense and raise the question of what the clinician should do when histologically normal margins harbor genetic alterations. In addition, a significant limitation of these molecular analyses of surgical margins is the considerable time required to perform them, which significantly limits their intraoperative usefulness. Unlike direct visualization techniques using vital dyes or autofluorescence, molecular genetic analysis does not provide instantaneous results. Nonetheless, automation and streamlining of methodologies is dramatically decreasing turnaround times, nearing the point where intraoperative genetic analysis of surgical margins may become feasible some future date. ${ }^{62,66}$

At this time, targeted molecular margin analysis remains in its infancy. As mentioned, the strategy of molecular margins addresses mucosal genetic alterations, whereas most inadequate margins are encountered in deep connective tissue planes. ${ }^{30}$ Furthermore, current definitions of cancer are primarily made histopathologically in most cases, with clinical correlation. Is it appropriate to render additional cancer therapy in a region of the head and neck in which the margins are normal by clinical and histologic examination but demonstrate genetic instability, particularly when even dysplasia, which is in principle a more advanced preneoplasia, is often deemed negative? Do the ends of crafting such a treatment plan justify the means in terms of morbidity and cost? These questions and others will need to remain at the forefront of our investigation as we pursue a molecular diagnosis of HNSCC.

\section{Management of positive margins}

There seems to be little consensus on the treatment of positive margins other than perhaps avoiding them in the first place. For tumors from oral and oropharyngeal sites that are accessible for open or transoral surgery and excised with inadequate final margins, many surgeons will opt to reexcise tissue if feasible, especially if this would abrogate the need for adjuvant radiotherapy. For inadequate laryngeal margins, there is no consensus about whether a "second-look" procedure with repeat excisional biopsy, 4 to 6 weeks after initial surgery, is better than a "watch-and-wait" approach with close clinical followup. ${ }^{18,19,22}$ Retrospective studies seem to imply that either approach is legitimate. It is common to find no residual carcinoma in margin reexcisions, which does not invalidate this approach.

There has been recent interest in adding chemotherapy to the adjuvant radiation in patients at increased risk of locoregional failure. ${ }^{67}$ A small study with just 51 patients, radiation therapy oncology group (RTOG) 88-24, found that the addition of chemotherapy helped to increase locoregional control over postoperative radiotherapy alone. Interestingly, half of the patients in this study entered with positive margins. ${ }^{68}$ Two large prospective studies randomizing patients at high risk to postoperative radiotherapy with or without concurrent chemotherapy showed a significant decrease in both local and regional failure in the group who received concurrent chemotherapy. The definition of high-risk factors included positive margins - although that was defined as "less than $5 \mathrm{~mm}$ " in the European Organisation for Research and Treatment of Cancer (EORTC) study and "at the surgical margin "in the RTOG study. ${ }^{69,70}$ Nevertheless, when the data from both studies with both definitions were combined, these patients (along with those with extracapsular extension) treated with postoperative chemoradiotherapy had a $42 \%$ reduction in locoregional failure and a significant improvement in overall survival rates. ${ }^{67} \mathrm{~A}$ final noteworthy but less commonly used modality that has the potential to improve local control in cases of close or positive margins is adjuvant $\mathrm{I}^{125}$ brachytherapy in conjunction 
with external radiotherapy. ${ }^{71}$ Because the chance of recurrence is not only related to margin status but also to grading features, ${ }^{12,56}$ an additional question is whether factors such as perineural and lymphovascular invasion per se require additional treatment. These poor prognostic features are seldom quantified when limited to the interior of a specimen with clear margins. If additional treatment is chosen in those cases with unfavorable grading features, it may be argued that adjuvant radiotherapy is more likely to adequately deal with this increased risk on recurrence than additional surgery given the growth pattern of these tumors. However, data on adjuvant treatment in the postoperative setting in relation to features of the primary tumor are limited and results from randomized trials are needed to answer these questions, and parameters such as growth pattern should be considered in the context of other risk factors.

The last consideration is one that has not been fully explored, and that is the effect of field cancerization on margin status. If there truly is a dramatic field effect, reexcising a margin for the presence of dysplasia may be futile, because there is presumably "at-risk" mucosa in the entire vicinity of the tumor. Most experienced surgeons have at one time or another encountered a patient with severe dysplasia or in-situ carcinoma widely involving the upper aerodigestive tract. There is no literature to clinically guide a surgeon who is striving for an adequate margin in the setting of field cancerization.

\section{CONCLUSIONS}

Inadequate surgical resection margins contribute to increased local recurrence and morbidity rates, decreased survival rates, and increased cost to society. It might commit patients to adjuvant radiotherapy, which may not have been otherwise necessary. Therefore surgeons need to strive to achieve adequate resection margins whenever possible. Currently, it is not possible to compare outcomes on the basis of margins between institutions given the disparate practice patterns, institution and surgical biases, diverse surgical instrumentations, and the varied resection techniques currently used. Currently there is no uniformity in the definitions of adequate surgical margins. However, we do recommend that margin distance be measured in millimeters and recorded on the surgical pathology report. For carcinomas of the glottic larynx, narrow margins of just 1 to $2 \mathrm{~mm}$ seem adequate. Within the oral cavity $5 \mathrm{~mm}$ represents the most commonly used margin standard. For carcinomas of the supraglottis, hypopharynx, and oropharynx, precise ideal routine metrics still need to be defined.

Positive margins should be surgically cleared whenever possible. If this is not feasible, chemoradiotherapy is superior to adjuvant radiotherapy alone. Other tumor features such as lymphovascular invasion, perineural spread, and high-risk status are also involved in the risk assessment for local recurrence and impact the decision for adjuvant treatment. An ongoing awareness and assessment of tumor factors including molecular markers need to be pursued from a research standpoint, with clinical correlation. Future studies need to specifically report local control and regional control rates, as well as overall survival. An awareness of the operator dependence regarding surgi- cal margin assessment requires ongoing review and improvement to obtain the best outcomes. Future research should begin with authors clearly defining their respective institutional definitions. Head and neck surgeons and pathologists should work together toward standardizing margin assessment from a multidisciplinary and multiinstitutional perspective.

\section{REFERENCES}

1. Jesse RH, Sugarbaker EV. Squamous cell carcinoma of the oropharynx: why we fail. Am J Surg 1976;132:435-438.

2. Meier JD, Oliver DA, Varvares MA. Surgical margin determination in head and neck oncology: current clinical practice. The results of an International American Head and Neck Society Member Survey. Head Neck 2005;27:952-958.

3. Looser KG, Shah JP, Strong EW. The significance of "positive" margins in surgically resected epidermoid carcinomas. Head Neck 1978;1: 107-111.

4. Chen TY, Emrich LJ, Driscoll DL. The clinical significance of pathological findings in surgically resected margins of the primary tumor in head and neck carcinoma. Int J Radiat Oncol Biol Phys 1987;13:833-837.

5. Loree TR, Strong EW. Significance of positive margins in oral cavity squamous carcinoma. Am J Surg 1990;160:410-414.

6. Garzino-Demo P, Dell'Acqua A, Dalmasso P, et al. Clinicopathologic parameters and outcome of 245 patients operated for oral squamous cell carcinoma. J Craniomaxillofac Surg 2006;34:344-350.

7. El-Husseiny G, Kandil A, Jamshed A, et al. Squamous cell carcinoma of the oral tongue: an analysis of prognostic factors. Br J Oral Maxillofac Surg 2000;38:193-199.

8. Amaral TMP, Freire ARS, Carvalho AL, et al. Predictive factors of occult metastasis and prognosis of clinical stages I and II squamous cell carcinoma of the tongue and floor of mouth. Oral Oncol 2004;40:780-786.

9. Weijers M, Snow GB, Bezemer DP, et al. The status of the deep surgical margins in tongue and floor of mouth squamous cell carcinoma and risk of local recurrence: an analysis of 68 patients. Int J Oral Maxillofac Surg 2004;33:146-149.

10. Liao CT, Chang JT, Wang HM, et al. Analysis of risk factors of predictive local tumor control in oral cavity cancer. Ann Surg Oncol 2008;15: 915-922.

11. Kademani D, Bell RB, Bagheri S, et al. Prognostic factors in intraoral squamous cell carcinoma: the influence of histologic grade. J Oral Maxillofac Surg 2005;63:1599-1605.

12. Brandwein-Gensler M, Teixeira MS, Lewis CM, et al. Oral squamous cell carcinoma: histologic risk assessment, but not margin status, is strongly predictive of local disease-free and overall survival. Am J Surg Pathol 2005;29:167-178.

13. McMahon J, O'Brien CJ, Pathak I, et al. Influence of condition of surgical margins on local recurrence and disease-specific survival in oral and oropharyngeal cancer. Br J Oral Maxillofac Surg 2003;41:224-231.

14. de Visscher JGAM, Gooris PJJ, Vermey A, Roodenburg JLN. Surgical margins for resection of squamous cell carcinoma of the lower lip. Int $J$ Oral Maxillofac Surg 2002;31:154-157.

15. Sutton DN, Brown JS, Rogers SN, Vaughan ED, Woolgar JA. The prognostic implications of the surgical margin in oral squamous cell carcinoma. Int J Oral Maxillofac Surg 2003;32:30-34.

16. Nakayama M, Holsinger C, Okamoto $M$, et al. Clinicopathological analyses of fifty supracricoid laryngectomized specimens: evidence base supporting minimal margins. ORL J Otorhinolaryngol Relat Spec 2009;71: 305-311.

17. Ossoff RH, Sisson GA, Shapshay SM. Endoscopic management of selected early vocal cord carcinoma. Ann Otol Rhinol Laryngol 1985;94: 560-564.

18. Grant DG, Bradley PT, Parmar A, et al. Implications of positive margins or incomplete excision in laryngeal cancer treated by transoral laser microsurgery: how we do it. Clin Otolaryngol 2009;34:485-489.

19. Preuss SF, Cramer K, Drebber U, et al. Second-look microlaryngoscopy to detect residual carcinoma in patients after laser surgery for T1 and T2 laryngeal cancer. Acta Otolaryngol 2009;129:881-885.

20. Brøndbo K, Fridrich K, Boysen M. Laser surgery of T1a glottic carcinomas; significance of resection margins. Eur Arch Otorhinolaryngol 2007; 264:627-630.

21. Sigston E, de Mones E, Babin E, et al. Early-stage glottic cancer: oncological results and margins in laser cordectomy. Arch Otolaryngol Head Neck Surg 2006;132:147-152.

22. Hartl DM, de Monès E, Hans S, et al. Treatment of early-stage glottic cancer by transoral laser resection. Ann Otol Rhinol Laryngol 2007;116: 832-836.

23. Peretti G, Piazza C, Cocco D, et al. Transoral CO(2) laser treatment for T(is)-T(3) glottic cancer: the University of Brescia experience on 595 patients. Head Neck 2010;32:977-983. 
24. Crespo AN, Chone CT, Gripp FM, et al. Role of margin status in recurrence after $\mathrm{CO} 2$ laser endoscopic resection of early glottic cancer. Acta Otolaryngol 2006;126:306-310.

25. Blanch JL, Vilaseca I, Caballero M, et al. Outcome of transoral laser microsurgery for T2-T3 tumors growing in the laryngeal anterior commissure. Head Neck 2011;33:1252-1259.

26. Spector JG, Sessions DG, Lenox J, Simpson J. Management of T3N1 glottic carcinoma: therapeutic outcomes. Laryngoscope 2006;116:106-110.

27. Gallo A, Manciocco V, Simonelli M, et al. Supracricoid partial laryngectomy in the treatment of laryngeal cancer: univariate and multivariate analysis of prognostic factors. Arch Otolaryngol Head Neck Surg 2005 131:620-625.

28. Bradford CR, Wolf GT, Fisher SG, McClatchey KD. Prognostic importance of surgical margins in advanced laryngeal squamous carcinoma. Head Neck 1996;18:11-16.

29. Sessions DG, Lenox J, Spector GJ. Supraglottic laryngeal cancer: analysis of treatment results. Laryngoscope 2005;115:1402-1410.

30. Woolgar JA, Triantafyllou A. A histopathological appraisal of surgical margins in oral and oropharyngeal cancer resection specimens. Oral Oncol 2005;41:1034-1043.

31. Black C, Marotti J, Zarovnaya E, Paydarfar J. Critical evaluation of frozen section margins in head and neck cancer resections. Cancer 2006;107: 2792-2800.

32. Remacle M, Matar N, Delos M, Nollevaux MC, Jamart J, Lawson G. Is frozen section reliable in transoral $\mathrm{CO}(2)$ laser-assisted cordectomies? Eur Arch Otorhinolaryngol 2010;267:397-400.

33. Kerawala CJ, Ong TK. Relocating the site of frozen sections-is there room for improvement? Head Neck 2001;23:230-232.

34. Olson SM, Hussaini M, Lewis JS Jr. Frozen section analysis of margins for head and neck tumor resections: reduction of sampling errors with a third histologic level. Mod Pathol 2011;24:665-670.

35. Cooley ML, Hoffman HT, Robinson RA. Discrepancies in frozen section mucosal margin tissue in laryngeal squamous cell carcinoma. Head Neck 2002;24:262-267.

36. Jäckel MC, Ambrosch P, Martin A, Steiner W. Impact of re-resection for inadequate margins on the prognosis of upper aerodigestive tract cancer treated by laser microsurgery. Laryngoscope 2007;117:35-36.

37. Steiner W, Ambrosch P, Hess CF, Kron M. Organ preservation by transoral laser microsurgery in piriform sinus carcinoma. Otolaryngol Head Neck Surg 2001;124:58-67.

38. Steiner W, Fierek O, Ambrosch P, Hommerich CP, Kron M. Transoral laser microsurgery for squamous cell carcinoma of the base of the tongue. Arch Otolaryngol Head Neck Surg 2003;129:36-43.

39. Johnson RE, Sigman JD, Funk GF, Robinson RA, Hoffman HT. Quantification of surgical margin shrinkage in the oral cavity. Head Neck 1997;19: 281-286.

40. Mistry RC, Qureshi SS, Kumaran C. Post-resection mucosal margin shrinkage in oral cancer: quantification and significance. J Surg Oncol 2005;91:131-133

41. Forrest LA, Schuller DE, Lucas JG, Sullivan MJ. Rapid analysis of mandibular margins. Laryngoscope 1995;105:475-477.

42. Mahmood S, Conway DI, Ramesar K. Use of intra-operative cytological assessment of mandibular marrow scrapings to predict resection margin status in patients with squamous cell carcinoma. J Oral Maxillofac Surg 2001;59:1138-1141.

43. Oxford LE, Ducic Y. Intraoperative evaluation of cortical bony margins with frozen-section analysis. Otolaryngol Head Neck Surg 2006;134 $138-141$

44. Wysluch A, Stricker I, Holzle F, Wolff K-D, Maurer P. Intraoperative evaluation of bony margins with frozen-section analysis and trephine drill extraction techqnique: a preliminary study. Head Neck 2010;32 1473-1478.

45. Weisberger EC, Hilburn M, Johnson B, Nguyen C. Intraoperative microwave processing of bone margins during resection of head and neck cancer. Arch Otolaryngol Head Neck Surg 2001;127:790-793.

46. Jerjes W, Swinson B, Johnson KS, Thomas GJ, Hopper C. Assessment of bony resection margins in oral cancer using elastic scattering spectroscopy: a study on archival material. Arch Oral Biol 2005;50:361-366.

47. Cikojevíc D, Gluncíc I, Pesutíc-Pisac V. Comparison of contact endoscopy and frozen section histopathology in the intra-operative diagnosis of laryngeal pathology. J Laryngol Otol 2008;122:836-839.

48. Dedivitis RA, Pfuetzenreiter EG Jr. Guimarães AV. Contact endoscopy of the larynx as an auxiliary method to the surgical margins in frontolateral laryngectomy. Acta Otorhinolaryngol Ital 2009;29:16-20.
49. Keereweer S, Sterenborg HJ, Kerrebijn JD, Van Driel PB, de Jong RJ, Löwik CW. Image-guided surgery in head and neck cancer: current practice and future directions of optical imaging. Head Neck 2012;34: $120-126$.

50. McMahon JD, Devine JC, Hetherington J, et al. Involved surgical margins in oral and oropharyngeal carcinoma - an anatomical problem? $\mathrm{Br} \mathrm{J}$ Oral Maxillofac Surg 2011;49:172-175.

51. Kreeft AM, Smeele LE, Rasch CRN, et al. Preoperative imaging and surgical margins in maxillectomy patients. Head Neck 2012 Jan 17.doi; 10.1002/hed. 21987. [Epub ahead of print] PubMed PMID: 22252962.

52. Broders AC. Squamous-cell epithelioma of the lip: a study of five hundred and thirty-seven cases. JAMA 1920;74:656-664.

53. Sparano A, Weinstein G, Chalian A, Yodul M, Weber R. Multivariate predictors of occult neck metastasis in early oral tongue cancer. Otolaryngol Head Neck Surg 2004;131:472-476.

54. Anneroth G, Hansen LS. A methodologic study of histologic classification and grading of malignancy in oral squamous cell carcinoma. Scand J Dent Res 1984;92:448-468.

55. Bryne M, Koppang HS, Lilleng R, Stene T, Bang G, Dabelsteen E. New malignancy grading is a better prognostic indicator than Broders' grading in oral squamous cell carcinomas. J Oral Pathol Med 1989;18:432-437.

56. Brandwein-Gensler M, Smith RV, Wang B, et al. Validation of the histologic risk model in a new cohort of patients with head and neck squamous cell carcinoma. Am J Surg Pathol 2010;34:676-688.

57. Slaughter DP, Southwick HW, Smejkal W. "Field cancerization" in oral stratified squamous epithelium; clinical implications of multicentric origin. Cancer 1953;6:963-968.

58. Westra WH, Sidransky D. Phenotypic and genotypic disparity in premalignant lesions: of calm water and crocodiles. J Natl Cancer Inst 1998;90: $1500-1501$.

59. van Houten VM, Leemans CR, Kummer JA, et al. Molecular diagnosis of surgical margins and local recurrence in head and neck cancer patients: a prospective study. Clin Cancer Res 2004;10:3614-3620.

60. Sardi I, Franchi A, Ferriero G, et al. Prediction of recurrence by microsatellite analysis in head and neck cancer. Genes Chromosomes Cancer 2000;29:201-206.

61. Martone T, Gillio-Tos A, De Marco L, et al. Association between hypermethylated tumor and paired surgical margins in head and neck squamous cell carcinomas. Clin Cancer Res 2007;13:5089-5094.

62. Goldenberg D, Harden S, Masayesva BG, et al. Intraoperative molecular margin analysis in head and neck cancer. Arch Otolaryngol Head Neck Surg 2004;130:39-44.

63. Nathan CO, Liu L, Li BD, Abreo FW, Nandy I, De Benedetti A. Detection of the proto-oncogene eIF4E in surgical margins may predict recurrence in head and neck cancer. Oncogene 1997;15:579-584.

64. Dasgupta S, Koch R, Westra WH, et al. Mitochondrial DNA mutation in normal margins and tumors of recurrent head and neck squamous cell carcinoma patients. Cancer Prev Res (Phila) 2010;3:1205-1211.

65. Begum S, Cao D, Gillison M, Zahurak M, Westra WH. Tissue distribution of human papillomavirus 16 DNA integration in patients with tonsillar carcinoma. Clin Cancer Res 2005;11:5694-5699.

66. Harden SV, Thomas DC, Benoit N, et al. Real-time gap ligase chain reaction: a rapid semiquantitative assay for detecting p53 mutation at low levels in surgical margins and lymph nodes from resected lung and head and neck tumors. Clin Cancer Res 2004;10:2379-2385.

67. Bernier J, Cooper JS, Pajak TF, et al. Defining risk levels in locally advanced head and neck cancers: a comparative analysis of concurrent postoperative radiation plus chemotherapy trials of the EORTC (\#22931) and RTOG (\# 9501). Head Neck 2005;27:843-850.

68. Al-Sarraf M, Pajak TF, Byhardt RW, Beitler JJ, Salter MM, Cooper JS. Postoperative radiotherapy with concurrent cisplatin appears to improve locoregional control of advanced, resectable head and neck cancers: RTOG 88-24. Int J Radiat Oncol Biol Phys 1997;37:777-782.

69. Cooper JS, Pajak TF, Forastiere AA, et al. Postoperative concurrent radiotherapy and chemotherapy for high risk squamous cell carcinoma of the head and neck. N Engl J Med 2004;350:1937-1944.

70. Bernier J, Domenge C, Ozahin M et al. Postoperative irradiation with or without concomitant chemotherapy for locally advanced head and neck cancer. N Engl J Med 2004;350:1945-1952.

71. Beitler JJ, Smith RV, Silver CE, et al. Close or positive margins after surgical resection for the head and neck cancer patient: the addition of brachytherapy improves local control. Int J Radiat Oncol Biol Phys 1998; 40:313-317. 